\title{
Assessment of Spring Defoliation to Improve Fall Forage Quality of Bluebunch Wheatgrass (Agropyron spicatum)
}

MICHAEL D. PITT

\section{Abstract}

Bluebunch wheatgrass (Agropyron spicatum (Pursh) Scribn. \& Smith) was clipped at 4 phenological stages to compare forage quality of subsequent regrowth relative to nondefollated plants. Following 2 years of treatment, plants clipped at boot, emergence, nowering, and seed formation produced lower levels of ADF and higher values of $C P$ and $P$ than control plants at equivalent phenological stages. Clipping at boot and emergence for 2 years delayed flowering by 16 and 15 days, respectively, while subsequent flowering of plants clipped at flowering and seed formation occurred only sporadically. These delays in plant phenology altered forage quality on 26 October compared to nondefoliated plants. Clipping at boot, emergence, flowering, and seed formation reduced percent foliar ADF, while increasing relative proportions of $\mathrm{CP}, \mathrm{Ca}$, and $\mathrm{P}$ compared to untreated herbage. Crude protein in plants clipped for 2 years at emergence, flowering, and seed formation averaged $11.9 \%, 12.5 \%$, and $13.7 \%$, respectively. Phosphorus in regrowth foliage of plants clipped at flowering and seed formation equalled $0.22 \%$ and $0.26 \%$, respectively, on 26 October. These values exceed maintenance requirements of cattle and clk, indicating that judicious grazing management can improve nutritive values of bunchgrass vegetation.

Controlled livestock grazing has been recommended to improve or maintain both the quantity and quality of range forage (Stoddard 1946, Hyder and Sneva 1963, Hormay 1970, Hyder 1971, Jensen et al. 1972, Malachek et al. 1978). Anderson and Scherzinger (1975) summarized a grazing management plan for bluebunch wheatgrass/Idaho fescue (Agropyron spicatum (Pursh) Scribn. \& Smith/Festuca idahoensis Torr.) ranges of central Oregon which controls the intensity and season of livestock grazing in order to improve forage quality for wintering elk. This grazing management plan was based upon the theoretical assumption that spring grazing by livestock delayed plant phenology, such that nutrients were retained in the foliar portions of bunchgrasses rather than being translocated to the roots prior to the onset of summer aestivation (Anderson and Scherzinger 1975).

Many grazing management plans in British Columbia have been developed in response to the recommendations of Anderson and Scherzinger (1975), who cautioned, however, that their conclusions were predicated primarily upon biological principles combined with field observations. Although bluebunch wheatgrass has been studied intensively (Hanson and Stoddard 1940, Mcllvanie 1942, Stoddart 1946, Blaisdell and Pechanec 1949, Heady 1959, Mueggler 1975, Rickard et al. 1975, Uresk and Cline 1976, McLean and Wikeem 1985) virtually no quantitative data are available which describe the functional relationships among spring grazing, delays in plant phenology and resultant fall or winter forage quality. The objectives of this investigation were to document such relationships in order to assess the recommendations of Anderson and Schezinger (1975), particularly in relation to the most judicious phenological stage at which bluebunch wheatgrass should be grazed by cattle in order to improve fall forage quality for wildlife.

\footnotetext{
The author is associate professor, Department of Plant Science, University of British Columbia, Vancouver, Canada V6T 2A2.

Financial support for this research was provided by Agriculture Canada and the Natural Sciences and Engineering Research Council of Canada.

Manuscript accepted 14 July 1985
}

\section{Materials and Methods}

The research was conducted during 1978 and 1979 at the University of British Columbia in Vancouver. Experimental plots occurred at an elevation of $93 \mathrm{~m}$ on soils classified as Humo Ferric Podzols (Canada Soil Survey Committee 1978). The maritime climate is characterized by warm summers and cool winters, with average annual precipitation equal to $102 \mathrm{~cm}$ and an average frost free period of 210 days (Harry and Wright 1967).

Two hundred seeds of bluebunch wheatgrass were germinated in the greenhouse and transplanted to the experimental plots, at 1-meter centers, in August of 1976. In 1978 surviving plants were randomly assigned to each of 5 clipping treatments: control, plus herbage removal at boot, emergence, flowering, and seed formation stages of development. Moderate grazing was simulated by clipping plants to a $15-\mathrm{cm}$ stubble height.

Forty-five plants were allocated as control, so that 5 replicates were available for sampling at 9 phenological stages identified by Metcalfe (1973): vegetative, boot, emergence, flowering, seed formation, ripe seed, seed shatter, stem-cured, and weathered. Similarly, at each treatment stage of herbage removal, a sufficient number of plants were clipped so that 5 replicates could be harvested for chemical analysis at each subsequent stage of phenological development. This experimental approach permitted assessment of forage quality in regrowth herbage relative to forage quality in unclipped plants at comparable phenological stages. These procedures were repeated in 1979.

Forage samples were dried at $65^{\circ} \mathrm{C}$ for 24 hours, and then ground in a Wiley Mill through a 40 -mesh screen. Chemical analyses were conducted according to the procedures outlined by the Association of Official Analytical Chemists (1975). Forage digestibility was evaluated with acid detergent fiber (ADF), while nitrogen $(N)$, calcium $(\mathrm{Ca})$, and phosphorus $(\mathrm{P})$ were measured as assessments of forage quality. All chemical determinations were expressed as a percentage of dry matter. Percent crude protein (CP) was derived by multiplying percent nitrogen by 6.25 .

Differences in forage quality at equivalent phenological stages were determined with analysis of variance followed by a studentized Newman-Keuhls multiple range test at the 5\% level of significance (Steele and Torrie 1980). These analyses are presented for comparison with previous research on bluebunch wheatgrass, which typically reports forage quality at specific phenological stages.

The primary objective of the current study, to assess the potential of spring cattle grazing for improving forage quality, requires that comparisons also be made with respect to calendar date. Because of variabilities in plant maturity rates, differences in forage quality at phenological stages are not identical to differences in forage quality at specific calendar dates. Consequently, polynomial regressions were used to predict forage quality means for nine 25-day intervals beginning with 9 April and ending on 26 October. Regression equations were based upon forage quality measurements within each clipping treatment at each phenological stage. The predictor variable was measured as the number of days elapsed between 1 January and date of forage quality sampling.

The form of polynomial regressions varied among treatment means, and was selected to yield the highest coefficient of determi- 
nation for each regression equation. Predicted means are presented only for significant $(p<0.05)$ polynomial regressions at calendar dates when plants were sampled within each clipping treatment. Comparisons within calendar dates are indicated with $95 \%$ confidence intervals for each predicted mean.

\section{Results}

All clipping treatments delayed phenological development during both years of study. Plants defoliated at boot flowered an average of 9 and 16 days later than control plants in 1978 and 1979 , respectively. Equivalent delays occurred for plants clipped at emergence, which flowered approximately 15 days later than untreated plants in both 1978 and 1979. Subsequent flowering for plants clipped at flowering occurred sporadically, and averaged 73 and 194 days later than unclipped plants in 1978 and 1979, respectively. Flowering of plants clipped during seed formation averaged 95 and 142 days later than nondefoliated plants in 1978 and 1979 , respectively. During both years of study, clipped plants failed to reach the weathered stage of plant maturity.

These defoliation-induced delays in plant phenology form the basis for Anderson and Scherzinger's (1975) recommendation to utilize spring cattle grazing to enhance fall forage quality. For example, plants clipped at boot, emergence, and flowering in 1978 showed initial reductions in percent ADF in subsequent stages of plant phenology compared to unclipped plants (Table 1). These

Table 1. Acid detergent fiber $(\%)$ in regrowth of bluebunch wheatgrass.

\begin{tabular}{|c|c|c|c|c|c|}
\hline \multirow[b]{2}{*}{$\begin{array}{l}\text { Phenological } \\
\text { stage }\end{array}$} & \multicolumn{5}{|c|}{ Clipping treatment } \\
\hline & Control & Boot & $\begin{array}{l}\text { Emer- } \\
\text { gence }\end{array}$ & $\begin{array}{l}\text { Flower- } \\
\text { ing }\end{array}$ & $\begin{array}{c}\text { Seed } \\
\text { formation }\end{array}$ \\
\hline $\begin{array}{c}\text { Vegetative } \\
1978 \\
1979\end{array}$ & $\begin{array}{l}24.0 \\
33.8\end{array}$ & & & & \\
\hline $\begin{array}{l}\text { Boot } \\
1978 \\
1979\end{array}$ & $\begin{array}{l}32.6 \\
34.2\end{array}$ & & & & \\
\hline $\begin{array}{l}\text { Emergence } \\
1978 \\
1979\end{array}$ & $\begin{array}{l}37.4 \mathrm{a}^{1} \\
38.1 \mathrm{a}\end{array}$ & $\begin{array}{l}34.6 \mathrm{a} \\
30.7 \mathrm{~b}\end{array}$ & & & \\
\hline $\begin{array}{l}\text { Flowering } \\
1978 \\
1979\end{array}$ & $\begin{array}{l}38.4 \mathrm{ab} \\
39.3 \mathrm{a}\end{array}$ & $\begin{array}{l}39.5 b \\
33.9 b\end{array}$ & $\begin{array}{l}34.8 \mathrm{a} \\
33.8 \mathrm{~b}\end{array}$ & & \\
\hline $\begin{array}{l}\text { Seed Formation } \\
1978 \\
1979\end{array}$ & $\begin{array}{l}41.7 a \\
41.4 a\end{array}$ & $\begin{array}{l}34.8 \mathrm{~b} \\
34.2 \mathrm{~b}\end{array}$ & $\begin{array}{l}35.7 \mathrm{~b} \\
37.5 \mathrm{~b}\end{array}$ & $\begin{array}{l}38.6 \mathrm{ab} \\
39.0 \mathrm{~b}\end{array}$ & \\
\hline $\begin{array}{l}\text { Ripe Seed } \\
1978 \\
1979\end{array}$ & $\begin{array}{l}42.1 a \\
46.2 a\end{array}$ & $\begin{array}{l}38.3 b \\
37.8 b\end{array}$ & $\begin{array}{l}38.2 \mathrm{~b} \\
37.0 \mathrm{~b}\end{array}$ & $\begin{array}{l}41.3 \mathrm{ab} \\
35.8 \mathrm{~b}\end{array}$ & $39.8 \mathrm{ab}$ \\
\hline $\begin{array}{l}\text { Seed Shatter } \\
1978 \\
1979\end{array}$ & $\begin{array}{l}46.9 a \\
47.3 a\end{array}$ & $\begin{array}{l}43.6 \mathrm{a} \\
42.4 \mathrm{ab}\end{array}$ & $\begin{array}{l}43.5 \mathrm{a} \\
43.5 \mathrm{ab}\end{array}$ & $\begin{array}{l}39.8 \mathrm{~b} \\
36.4 \mathrm{ab}\end{array}$ & $\begin{array}{l}38.6 \mathrm{~b} \\
35.6 \mathrm{~b}\end{array}$ \\
\hline $\begin{array}{l}\text { Stem-cured } \\
1978 \\
1979\end{array}$ & $\begin{array}{l}43.2 \mathrm{a} \\
49.8 \mathrm{a}\end{array}$ & $\begin{array}{l}44.6 a \\
43.3 b\end{array}$ & $\begin{array}{l}41.3 \mathrm{ab} \\
38.1 \mathrm{c}\end{array}$ & $\begin{array}{l}42.5 a \\
42.4 b\end{array}$ & $\begin{array}{l}37.9 b \\
38.9 c\end{array}$ \\
\hline $\begin{array}{l}\text { Weathered } \\
1978 \\
1979\end{array}$ & 52.0 & & & & \\
\hline
\end{tabular}

${ }^{1}$ Means within rows followed by different letters are significantly different at $p<0.05$.

increases in digestibility generally failed to persist, however, although plants clipped at seed formation displayed significantly lower ADF (37.9\%) compared to untreated plants $(43.2 \%)$ at the stem-cured stage of plant maturity. Crude protein in plants clipped at boot and emergence in 1978 produced no statistically significant changes in percent crude protein at subsequent phenological stages compared to untreated plants (Table 2). Clipping at flowering and seed formation, however, increased CP to $11.9 \%$ and $13.4 \%$ respec-
Table 2. Crude protein (\%) in regrowth of bluebunch wheatgrass.

\begin{tabular}{|c|c|c|c|c|c|}
\hline \multirow[b]{2}{*}{$\begin{array}{l}\text { Phenological } \\
\text { stage }\end{array}$} & \multicolumn{5}{|c|}{ Clipping treatment } \\
\hline & Control & Boot & $\begin{array}{l}\text { Emer- } \\
\text { gence }\end{array}$ & $\begin{array}{l}\text { Flower- } \\
\text { ing }\end{array}$ & $\begin{array}{c}\text { Seed } \\
\text { formation }\end{array}$ \\
\hline $\begin{array}{l}\text { Vegetative } \\
1978 \\
1979\end{array}$ & $\begin{array}{l}26.8 \\
16.6\end{array}$ & & & & \\
\hline $\begin{array}{l}\text { Boot } \\
1978 \\
1979\end{array}$ & $\begin{array}{l}18.1 \\
14.1\end{array}$ & & & & \\
\hline $\begin{array}{c}\text { Emergence } \\
1978 \\
1979\end{array}$ & $\begin{array}{l}14.3 \mathrm{a}^{1} \\
10.8 \mathrm{a}^{2}\end{array}$ & $\begin{array}{l}17.2 \mathrm{a} \\
15.6 \mathrm{~b}\end{array}$ & & & \\
\hline $\begin{array}{c}\text { Flowering } \\
1978 \\
1979\end{array}$ & $\begin{array}{r}11.8 \mathrm{a} \\
7.3 \mathrm{a}\end{array}$ & $\begin{array}{l}11.5 \mathrm{a} \\
11.7 \mathrm{~b}\end{array}$ & $\begin{array}{l}14.0 \mathrm{a} \\
12.7 \mathrm{~b}\end{array}$ & & \\
\hline $\begin{array}{l}\text { Seed Formation } \\
1978 \\
1979\end{array}$ & $\begin{array}{r}10.0 \mathrm{a} \\
5.3 \mathrm{a}\end{array}$ & $\begin{array}{r}11.2 \mathrm{a} \\
8.8 \mathrm{~b}\end{array}$ & $\begin{array}{l}10.5 \mathrm{a} \\
8.6 \mathrm{~b}\end{array}$ & $\begin{array}{l}11.0 \mathrm{a} \\
10.3 \mathrm{~b}\end{array}$ & \\
\hline $\begin{array}{l}\text { Ripe Seed } \\
1978 \\
1979\end{array}$ & $\begin{array}{l}8.2 \mathrm{a} \\
3.6 \mathrm{a}\end{array}$ & $\begin{array}{l}9.0 \mathrm{a} \\
7.1 \mathrm{~b}\end{array}$ & $\begin{array}{l}8.9 a \\
8.9 b\end{array}$ & $\begin{array}{r}8.5 \mathrm{a} \\
15.8 \mathrm{c}\end{array}$ & $12.4 \mathrm{~b}$ \\
\hline $\begin{array}{l}\text { Seed Shatter } \\
1978 \\
1979\end{array}$ & $\begin{array}{l}7.3 \mathrm{a} \\
5.0 \mathrm{a}\end{array}$ & $\begin{array}{l}7.8 \mathrm{a} \\
8.1 \mathrm{~b}\end{array}$ & $\begin{array}{l}9.8 \mathrm{a} \\
9.3 \mathrm{~b}\end{array}$ & $\begin{array}{l}12.7 \mathrm{~b} \\
13.2 \mathrm{c}\end{array}$ & $\begin{array}{l}12.9 \mathrm{~b} \\
15.2 \mathrm{c}\end{array}$ \\
\hline $\begin{array}{c}\text { Stem-cured } \\
1978 \\
1979\end{array}$ & $\begin{array}{l}8.9 a \\
4.6 a\end{array}$ & $\begin{array}{l}9.3 \mathrm{a} \\
7.6 \mathrm{~b}\end{array}$ & $\begin{array}{c}10.6 \mathrm{ab} \\
8.7 \mathrm{~b}\end{array}$ & $\begin{array}{r}11.9 b c \\
9.6 b c\end{array}$ & $\begin{array}{l}13.4 \mathrm{c} \\
12.3 \mathrm{c}\end{array}$ \\
\hline $\begin{array}{l}\text { Weathered } \\
1978 \\
1979\end{array}$ & 6.1 & & & & \\
\hline
\end{tabular}

${ }^{1}$ Means within rows followed by different letters are significantly different at $p<0.05$.

tively at the stem-cured stage compared to only $8.9 \%$ for unclipped plants.

In contrast to ADF and CP, the initial year of clipping in 1978 produced no statistical differences in percent $\mathrm{Ca}$ at comparable stages of plant phenology (Table 3). Percent foliar P of plants clipped in 1978 generally increased initially compared to nondefoliated plants (Table 4). As the foliage matured, however, these differences diminished, such that no differences in phosphorus among treatments existed by the ripe seed stage of plant phenology.

A second year of defoliation in 1979 produced more notable and persistent changes in forage quality of regrowth herbage. Plants clipped at boot, emergence, flowering, and seed formation all provided lower ADF than control plants. These reductions occurred in phenological stages immediately following defoliation, and were still evident at the stem-cured stage of plant development (Table 1). Repeated clipping in 1979 similarly promoted additional increases in percent $\mathbf{C P}$, as all defoliation treatments showed higher CP percentages relative to unclipped plants at comparable stages of plant phenology. Percent CP at the stem-cured stage of plant phenology was enhanced by progressively later clipping treatments, as plants defoliated at boot, emergence, flowering and seed formation averaged $7.6,8.7,9.6$, and $12.3 \%$, respectively, compared to only $4.6 \% \mathrm{CP}$ for untreated plants (Table 2).

In contrast to 1978 , all clipping treatments in 1979 increased $\mathrm{Ca}$ and $P$ relative to untreated plants at equivalent stages of plant phenology. Percent $\mathrm{Ca}$ at the stem-cured stage of maturity was most pronounced in plants clipped at boot $(0.66 \%)$ and emergence $(0.67 \%)$, declining successively with later clipping treatments at flowering $(0.42 \%)$ and seed formation $(0.40 \%$ ) (Table 3 ). Increased foliar $\mathbf{P}$, however, became more evident with successively later clipping treatments, as nondefoliated plants averaged only $0.07 \%$ $P$ at the stem-cured stage of development, while plants clipped at boot, emergence, flowering, and seed formation equalled $0.13,0.14$, 
Table 3. Calcium (\%) in regrowth of bluebunch wheat grass.

\begin{tabular}{|c|c|c|c|c|c|}
\hline \multirow[b]{2}{*}{$\begin{array}{l}\text { Phenological } \\
\text { stage }\end{array}$} & \multicolumn{5}{|c|}{ Clipping treatment } \\
\hline & Control & Boot & $\begin{array}{l}\text { Emer- } \\
\text { gence }\end{array}$ & $\begin{array}{l}\text { Flower- } \\
\text { ing }\end{array}$ & $\begin{array}{c}\text { Seed } \\
\text { formation }\end{array}$ \\
\hline $\begin{array}{l}\text { Vegetative } \\
1978 \\
1979\end{array}$ & $\begin{array}{l}0.54 \\
0.40\end{array}$ & & & & \\
\hline $\begin{array}{l}\text { Boot } \\
1978 \\
1979\end{array}$ & $\begin{array}{l}0.26 \\
0.20\end{array}$ & & & & \\
\hline $\begin{array}{c}\text { Emergence } \\
1978 \\
1979\end{array}$ & $\begin{array}{l}0.26 \mathrm{a}^{1} \\
0.18 \mathrm{a}^{2}\end{array}$ & $\begin{array}{l}0.26 \mathrm{a} \\
0.36 \mathrm{~b}\end{array}$ & & & \\
\hline $\begin{array}{c}\text { Flowering } \\
1978 \\
1979\end{array}$ & $\begin{array}{l}0.36 a \\
0.17 a\end{array}$ & $\begin{array}{l}0.32 \mathrm{a} \\
0.32 \mathrm{~b}\end{array}$ & $\begin{array}{l}0.28 \mathrm{a} \\
0.24 \mathrm{~b}\end{array}$ & & \\
\hline $\begin{array}{l}\text { Seed Formatic } \\
1978 \\
1979\end{array}$ & $\begin{array}{l}0.38 a \\
0.24 a\end{array}$ & $\begin{array}{l}0.42 a \\
0.45 b\end{array}$ & $\begin{array}{l}0.35 a \\
0.41 b\end{array}$ & $\begin{array}{l}0.39 \mathrm{a} \\
0.58 \mathrm{c}\end{array}$ & \\
\hline $\begin{array}{l}\text { Ripe Seed } \\
1978 \\
1979\end{array}$ & $\begin{array}{l}0.34 a \\
0.19 a\end{array}$ & $\begin{array}{l}0.45 a \\
0.54 b\end{array}$ & $\begin{array}{l}0.43 a \\
0.45 b\end{array}$ & $\begin{array}{l}0.47 a \\
0.82 c\end{array}$ & $0.43 a$ \\
\hline $\begin{array}{c}\text { Seed Shatter } \\
1978 \\
1979\end{array}$ & $\begin{array}{l}0.37 \mathrm{a} \\
0.30 \mathrm{a}\end{array}$ & $\begin{array}{l}0.38 \mathrm{a} \\
0.57 \mathrm{~b}\end{array}$ & $\begin{array}{l}0.49 \mathrm{a} \\
0.43 \mathrm{ab}\end{array}$ & $\begin{array}{l}0.45 \mathrm{a} \\
0.58 \mathrm{~b}\end{array}$ & $\begin{array}{l}0.48 \mathrm{a} \\
0.64 \mathrm{~b}\end{array}$ \\
\hline $\begin{array}{c}\text { Stem-cured } \\
1978 \\
1979\end{array}$ & $\begin{array}{l}0.39 \mathrm{a} \\
0.22 \mathrm{a}\end{array}$ & $\begin{array}{l}0.43 \mathrm{a} \\
0.66 \mathrm{~b}\end{array}$ & $\begin{array}{l}0.44 a \\
0.67 b\end{array}$ & $\begin{array}{l}0.37 a \\
0.42 c\end{array}$ & $\begin{array}{l}0.50 \mathrm{a} \\
0.40 \mathrm{c}\end{array}$ \\
\hline $\begin{array}{l}\text { Weathered } \\
1978 \\
1979\end{array}$ & 0.25 & & & & \\
\hline
\end{tabular}

IMeans within rows followed by different letters are significantly different at $p<0.05$.

0.18 , and $0.25 \% \mathrm{P}$ respectively (Table 4 ).

\section{Effect of Spring Defoliation on Fall Forage Quality}

These altered nutrient values at comparable phenological stages tended to correspond with improved forage quality for grazing animals and likely resulted from a combination of stimulated tillering following clipping, plus removal of mature herbage during the initial year of clipping. The potential for improved forage quality resulting from spring defoliation, however, must be assessed ultimately by comparisons at calendar dates rather than equivalent phenological stages. For example, ADF for unclipped plants averaged $42.7 \%$ on 6 September 1978 , which did not differ significantly from plants clipped either at boot $(43.8 \%)$, emergence, (41.3\%) flowering (39.8\%), or seed formation (40.0\%). Following a second defoliation in 1979, however, all clipping treatments significantly reduced ADF concentrations, indicating greater forage digestibility compared to unclipped plants. These reductions in ADF appeared immediately following clipping and persisted throughout the growing season. Nondefoliated plants on 26 October 1979 averaged $52.3 \%$ ADF, while plants clipped at emergence, flowering, and seed formation equalled only $41.7,38.7$, and 37.3\% ADF, respectively (Table 5).

Crude protein in nondefoliated plants declined from $23.2 \%$ on 9 April to $9.4 \%$ on 6 September 1978. Clipping at the boot stage of plant phenology produced an initial increase on 29 May from 12.8 to $18.0 \% \mathrm{CP}$, which then declined rapidly, such that no differences with control plants existed by 23 June. Clipping at emergence in 1978 produced similar initial increases in CP compared to control plants, followed by a subsequent decline to levels of CP similar to foliage in unclipped plants. Only those plants clipped at seed formation provided foliar CP (12.5\%) on 6 September that excecded statistically the $9.4 \% \mathrm{CP}$ in unclipped plants. These higher levels persisted at least until 26 October when foliage
Table 4. Phosphorus (\%) in regrowth of bluebunch wheatgrass.

\begin{tabular}{|c|c|c|c|c|c|}
\hline \multirow[b]{2}{*}{$\begin{array}{l}\text { Phenological } \\
\text { stage }\end{array}$} & \multicolumn{5}{|c|}{ Clipping treatment } \\
\hline & Control & Boot & $\begin{array}{l}\text { Emer- } \\
\text { gence }\end{array}$ & $\begin{array}{l}\text { Flower- } \\
\text { ing }\end{array}$ & $\begin{array}{c}\text { Seed } \\
\text { formation }\end{array}$ \\
\hline $\begin{array}{c}\text { Vegetative } \\
1978 \\
1979\end{array}$ & $\begin{array}{l}0.43 \\
0.24\end{array}$ & & & & \\
\hline $\begin{array}{l}\text { Boot } \\
1978 \\
1979\end{array}$ & $\begin{array}{l}0.28 \\
0.22\end{array}$ & & & & \\
\hline $\begin{array}{l}\text { Emergence } \\
1978 \\
1979\end{array}$ & $\begin{array}{l}0.27 \mathrm{a}^{1} \\
0.16 \mathrm{a}^{2}\end{array}$ & $\begin{array}{l}0.34 b \\
0.23 b\end{array}$ & & & \\
\hline $\begin{array}{c}\text { Flowering } \\
1978 \\
1979\end{array}$ & $\begin{array}{l}0.21 \mathrm{a} \\
0.14 \mathrm{a}\end{array}$ & $\begin{array}{l}0.20 \mathrm{a} \\
0.18 \mathrm{a}\end{array}$ & $\begin{array}{l}0.23 \mathrm{~b} \\
0.23 \mathrm{a}\end{array}$ & & \\
\hline $\begin{array}{l}\text { Seed Formatio } \\
1978 \\
1979\end{array}$ & $\begin{array}{l}0.20 \mathrm{a} \\
0.10 \mathrm{a}\end{array}$ & $\begin{array}{l}0.22 \mathrm{a} \\
0.15 \mathrm{ab}\end{array}$ & $\begin{array}{l}0.20 \mathrm{a} \\
0.20 \mathrm{bc}\end{array}$ & $\begin{array}{l}0.18 b \\
0.19 c\end{array}$ & \\
\hline $\begin{array}{c}\text { Ripe Seed } \\
1978 \\
1979\end{array}$ & $\begin{array}{l}0.15 a \\
0.08 a\end{array}$ & $\begin{array}{l}0.19 \mathrm{a} \\
0.16 \mathrm{~b}\end{array}$ & $\begin{array}{l}0.16 a \\
0.18 b\end{array}$ & $\begin{array}{l}0.16 \mathrm{a} \\
0.31 \mathrm{c}\end{array}$ & $0.18 \mathrm{a}$ \\
\hline $\begin{array}{l}\text { Seed Shatter } \\
1978 \\
1979\end{array}$ & $\begin{array}{l}0.15 \mathrm{a} \\
0.09 \mathrm{a}\end{array}$ & $\begin{array}{l}0.15 a \\
0.16 b\end{array}$ & $\begin{array}{l}0.18 \mathrm{a} \\
0.18 \mathrm{~b}\end{array}$ & $\begin{array}{l}0.20 \mathrm{a} \\
0.22 \mathrm{c}\end{array}$ & $\begin{array}{l}0.21 \mathrm{a} \\
0.26 \mathrm{c}\end{array}$ \\
\hline $\begin{array}{c}\text { Stem-cured } \\
1978 \\
1979\end{array}$ & $\begin{array}{l}0.15 a \\
0.07 a\end{array}$ & $\begin{array}{l}0.18 \mathrm{a} \\
0.13 \mathrm{~b}\end{array}$ & $\begin{array}{l}0.20 \mathrm{a} \\
0.14 \mathrm{~b}\end{array}$ & $\begin{array}{l}0.19 a \\
0.18 b\end{array}$ & $\begin{array}{l}0.21 \mathrm{a} \\
0.25 \mathrm{c}\end{array}$ \\
\hline $\begin{array}{l}\text { Weathered } \\
1978 \\
1979\end{array}$ & 0.09 & & & & \\
\hline
\end{tabular}

'Means within rows followed by different letters are significantly different at $p<0.05$.

clipped at flowering and seed formation in 1978 averaged 15.4 and $13.3 \% \mathrm{CP}$, respectively, compared to only $9.4 \%$ for nondefoliated plants on 6 September (Table 6).

Following repeated clipping in 1979, all treated plants produced statisticlly significant increases in percent $\mathrm{CP}$ compared to unclipped plants, which averaged only $5.6 \%$ on 26 October. Increases in CP were enhanced by successively later clipping regimes, as plants clipped at emergence, flowering, and seed formation averaged $11.9,12.5$, and $13.7 \% \mathrm{CP}$, respectively, on 26 October 1979. Plants clipped at boot in 1979 initially provided increased levels of CP, but failed to retain such increases, compared to control plants, beyond 6 September (Table 6).

Clipping in 1978 failed to produce statistically significant increases in calcium compared to untreated plants, which averaged $0.36 \% \mathrm{Ca}$ on 6 September. In 1979, however, foliar regrowth of clipped plants contained significantly more $\mathrm{Ca}$ than untreated plants. Predicted average Ca for control plants equalled only $0.19 \%$ on 26 October compared to $0.80,0.57$ and $0.52 \%$ for plants harvested initially at emergence, flowering, and seed formation, respectively (Table 7).

Percent phosphorus in clipped herbage displayed trends similar to those provided by calcium. Although clipping at boot in 1978 increased $P$ initially, from $0.24 \%$ to $0.36 \%$ on 29 May, no significant differences remained by 23 June. On 6 September, no treated plants differed statistically from control plants, which averaged $0.17 \%$ P (Table 8).

Following a second application of treatments in 1979, percent foliar $\mathbf{P}$ in regrowth of all clipped plants exceeded $\mathbf{P}$ contained in unclipped plants. Such differences persisted until 26 October when unclipped plants $(0.09 \%)$ contained significantly less $P$ than plants clipped at emergence $(0.14 \%)$, flowering $(0.22 \%)$ or seed formation $(0.26 \%)$ (Table 8$)$. 
Table 5. Predicted means and $95 \%$ confidence intervals for acid detergent fiber $(\%)$ in regrowth of bluebunch wheatgrass.

\begin{tabular}{|c|c|c|c|c|c|}
\hline & \multirow[b]{2}{*}{ Control } & \multicolumn{4}{|c|}{ Clipping treatment } \\
\hline & & Boot & Emergence & Flowering & Seed formation \\
\hline $\begin{array}{r}\text { April } 9 \\
1978 \\
1979\end{array}$ & $\begin{array}{l}23.8 \pm 3.3 \\
34.2 \pm 1.5\end{array}$ & & & & \\
\hline $\begin{array}{r}\text { May } 5 \\
1978 \\
1979\end{array}$ & $\begin{array}{l}33.7 \pm 1.0 \\
36.5 \pm 1.2\end{array}$ & & & & \\
\hline $\begin{array}{c}\text { May } 29 \\
1978 \\
1979\end{array}$ & $\begin{array}{l}39.0 \pm 1.1 \\
38.7 \pm 1.0\end{array}$ & $\begin{array}{l}34.1 \pm 3.0 \\
30.3 \pm 1.5\end{array}$ & & & \\
\hline $\begin{array}{c}\text { June } 23 \\
1978 \\
1979\end{array}$ & $39.8 \pm 1.0$ & $\begin{array}{l}37.4 \pm 1.6 \\
41.0 \pm 0.9\end{array}$ & $\begin{array}{l}33.1 \pm 1.9 \\
33.0 \pm 1.1\end{array}$ & & $32.8 \pm 2.3$ \\
\hline $\begin{array}{c}\text { July } 18 \\
1978 \\
1979\end{array}$ & $\begin{array}{l}41.3 \pm 1.0 \\
43.3 \pm 0.8\end{array}$ & $\begin{array}{l}34.3 \pm 2.2 \\
35.9 \pm 0.8\end{array}$ & $\begin{array}{l}35.8 \pm 1.3 \\
37.5 \pm 2.2\end{array}$ & & $39.9 \pm 0.9$ \\
\hline $\begin{array}{c}\text { August } 12 \\
1978 \\
1979\end{array}$ & $\begin{array}{l}45.4 \pm 1.1 \\
45.5 \pm 0.9\end{array}$ & $\begin{array}{l}41.7 \pm 1.6 \\
38.6 \pm 0.8\end{array}$ & $\begin{array}{l}38.6 \pm 1.0 \\
38.6 \pm 1.8\end{array}$ & $39.8 \pm 1.7$ & $40.4 \pm 1.2$ \\
\hline $\begin{array}{c}\text { September } \\
1978 \\
1979\end{array}$ & $\begin{array}{l}42.7 \pm 1.7 \\
47.8 \pm 1.1\end{array}$ & $\begin{array}{l}43.8 \pm 2.0 \\
41.4 \pm 1.0\end{array}$ & $\begin{array}{l}41.3 \pm 1.4 \\
38.1 \pm 1.2\end{array}$ & $39.8 \pm 4.1$ & $40.0 \pm 0.9$ \\
\hline $\begin{array}{c}\text { October I } \\
1978 \\
1979\end{array}$ & $50.1 \pm 1.3$ & $44.2 \pm 1.4$ & $\begin{array}{l}44.0 \pm 2.1 \\
38.4 \pm 1.9\end{array}$ & $42.5 \pm 8.6$ & $38.9 \pm 0.5$ \\
\hline $\begin{array}{c}\text { October } 26 \\
1978 \\
1979\end{array}$ & $52.3 \pm 1.6$ & & $41.7 \pm 2.3$ & $38.7 \pm 5.6$ & $\begin{array}{l}36.9 \pm 1.4 \\
37.3 \pm 3.4\end{array}$ \\
\hline
\end{tabular}

Table 6. Predicted means and $95 \%$ confidence intervals for crude protein (\%) in regrowth of bluebunch wheatgrass.

\begin{tabular}{|c|c|c|c|c|c|}
\hline & \multirow[b]{2}{*}{ Control } & \multicolumn{4}{|c|}{ Clipping treatment } \\
\hline & & Boot & Emergence & Flowering & Seed formation \\
\hline $\begin{array}{r}\text { April } 9 \\
1978 \\
1979\end{array}$ & $\begin{array}{l}23.2 \pm 2.3 \\
15.3 \pm 1.1\end{array}$ & & & & \\
\hline $\begin{array}{r}\text { May } 5 \\
1978 \\
1979\end{array}$ & $\begin{array}{l}16.7 \pm 0.7 \\
11.5 \pm 0.7\end{array}$ & & & & \\
\hline $\begin{array}{c}\text { May } 29 \\
1978 \\
1979\end{array}$ & $\begin{array}{r}12.8 \pm 0.7 \\
7.9 \pm 0.4\end{array}$ & $\begin{array}{l}18.0 \pm 2.2 \\
20.5 \pm 2.4\end{array}$ & & & \\
\hline $\begin{array}{c}\text { June } 23 \\
1978 \\
1979\end{array}$ & $\begin{array}{r}11.0 \pm 0.6 \\
5.2 \pm 0.6\end{array}$ & $\begin{array}{l}10.7 \pm 1.3 \\
11.8 \pm 0.9\end{array}$ & $\begin{array}{l}14.8 \pm 1.7 \\
11.0 \pm 1.3\end{array}$ & & \\
\hline $\begin{array}{c}\text { July } 18 \\
1978 \\
1979\end{array}$ & $\begin{array}{l}9.4 \pm 0.6 \\
3.9 \pm 0.6\end{array}$ & $\begin{array}{r}10.9 \pm 1.2 \\
7.8 \pm 0.9\end{array}$ & $\begin{array}{r}10.7 \pm 1.1 \\
8.9 \pm 0.8\end{array}$ & & $11.7 \pm 1.0$ \\
\hline $\begin{array}{c}\text { August } \\
1978 \\
1979\end{array}$ & $\begin{array}{l}7.4 \pm 0.8 \\
3.7 \pm 0.5\end{array}$ & $\begin{array}{l}9.1 \pm 1.2 \\
7.0 \pm 0.7\end{array}$ & $\begin{array}{l}8.9 \pm 1.2 \\
8.0 \pm 1.1\end{array}$ & $9.0 \pm 1.8$ & $12.1 \pm 0.7$ \\
\hline $\begin{array}{c}\text { Septemb } \\
1978 \\
1979\end{array}$ & $\begin{array}{l}9.4 \pm 1.1 \\
4.5 \pm 1.1\end{array}$ & $\begin{array}{l}8.4 \pm 1.3 \\
7.6 \pm 0.6\end{array}$ & $\begin{array}{l}9.3 \pm 1.2 \\
8.2 \pm 1.0\end{array}$ & $11.2 \pm 0.9$ & $12.5 \pm 0.6$ \\
\hline $\begin{array}{c}\text { October } \\
1978 \\
1979\end{array}$ & $5.5 \pm 1.4$ & $7.8 \pm 1.9$ & $\begin{array}{r}12.1 \pm 2.9 \\
9.5 \pm 0.8\end{array}$ & $13.3 \pm 1.9$ & $12.9 \pm 0.7$ \\
\hline $\begin{array}{c}\text { October } \\
1978 \\
1979\end{array}$ & $5.6 \pm 0.9$ & & $11.9 \pm 1.8$ & $\begin{array}{l}15.4 \pm 3.5 \\
12.5 \pm 3.0\end{array}$ & $\begin{array}{l}13.3 \pm 0.9 \\
13.7 \pm 3.1\end{array}$ \\
\hline
\end{tabular}


Table 7. Predicted means and $95 \%$ confidence intervals for calcium $(\%)$ in regrowth of bluebunch whentgrass.

\begin{tabular}{|c|c|c|c|c|c|}
\hline & \multirow[b]{2}{*}{ Control } & \multicolumn{4}{|c|}{ Clipping treatment } \\
\hline & & Boot & Emergence & Flowering & Seed formation \\
\hline $\begin{array}{r}\text { April } 9 \\
1978 \\
1979\end{array}$ & $\begin{array}{l}0.33 \pm .05 \\
0.38 \pm .08\end{array}$ & & & & \\
\hline $\begin{array}{r}\text { May } 5 \\
1978 \\
1979\end{array}$ & $\begin{array}{l}0.26 \pm .03 \\
0.25 \pm .03\end{array}$ & & & & \\
\hline $\begin{array}{c}\text { May } 29 \\
1978 \\
1979\end{array}$ & $\begin{array}{l}0.27 \pm .02 \\
0.16 \pm .03\end{array}$ & $\begin{array}{l}0.25 \pm .04 \\
0.30 \pm .08\end{array}$ & & & \\
\hline $\begin{array}{c}\text { June } 23 \\
1978 \\
1979\end{array}$ & $\begin{array}{l}0.31 \pm .03 \\
0.17 \pm .03\end{array}$ & $\begin{array}{l}0.35 \pm .03 \\
0.37 \pm .05\end{array}$ & $\begin{array}{l}0.29 \pm .06 \\
0.21 \pm .10\end{array}$ & & \\
\hline $\begin{array}{r}\text { July } 18 \\
1978 \\
1979\end{array}$ & $\begin{array}{l}0.37 \pm .03 \\
0.23 \pm .03\end{array}$ & $\begin{array}{l}0.41 \pm .03 \\
0.44 \pm .04\end{array}$ & $\begin{array}{l}0.35 \pm .04 \\
0.40 \pm .08\end{array}$ & & \\
\hline $\begin{array}{c}\text { August } 1 \\
1978 \\
1979\end{array}$ & $\begin{array}{l}0.39 \pm .03 \\
0.27 \pm .03\end{array}$ & $\begin{array}{l}0.43 \pm .03 \\
0.51 \pm .04\end{array}$ & $\begin{array}{l}0.41 \pm .03 \\
0.20 \pm .17\end{array}$ & $0.43 \pm .06$ & \\
\hline $\begin{array}{c}\text { Septemb } \\
1978 \\
1979\end{array}$ & $\begin{array}{l}0.36 \pm .06 \\
0.23 \pm .04\end{array}$ & $\begin{array}{l}0.41 \pm .04 \\
0.59 \pm .05\end{array}$ & $\begin{array}{l}0.46 \pm .05 \\
0.40 \pm .12\end{array}$ & $0.45 \pm 0.7$ & \\
\hline $\begin{array}{c}\text { October } \\
1978 \\
1979\end{array}$ & $0.14 \pm .09$ & $0.66 \pm .07$ & $\begin{array}{l}0.52 \pm .07 \\
0.80 \pm .02\end{array}$ & $0.37 \pm .07$ & $0.49 \pm .13$ \\
\hline $\begin{array}{c}\text { October } \\
1978 \\
1979\end{array}$ & $0.19 \pm .05$ & & $0.80 \pm .14$ & $0.57 \pm .17$ & $0.52 \pm .16$ \\
\hline
\end{tabular}

Table 8. Predicted means and $95 \%$ confidence intervals for phosphorus $(\%)$ in regrowth of bluebunch wheatgrass.

\begin{tabular}{|c|c|c|c|c|c|}
\hline & \multirow[b]{2}{*}{ Control } & \multicolumn{4}{|c|}{ Clipping treatment } \\
\hline & & Boot & Emergence & Flowering & Seed formation \\
\hline $\begin{array}{r}\text { April 9 } \\
1978 \\
1979\end{array}$ & $\begin{array}{l}0.33 \pm .02 \\
0.24 \pm .02\end{array}$ & & & & \\
\hline $\begin{array}{r}\text { May } 5 \\
1978 \\
1979\end{array}$ & $\begin{array}{l}0.28 \pm .01 \\
0.19 \pm .01\end{array}$ & & & & \\
\hline May 29 & & & & & \\
\hline $\begin{array}{l}1978 \\
1979\end{array}$ & $\begin{array}{l}0.24 \pm .01 \\
0.15 \pm .01\end{array}$ & $\begin{array}{l}0.36 \pm .04 \\
0.19 \pm .03\end{array}$ & & & \\
\hline $\begin{array}{c}\text { June } 23 \\
1978 \\
1979\end{array}$ & $\begin{array}{l}0.21 \pm .01 \\
0.12 \pm .01\end{array}$ & $\begin{array}{l}0.20 \pm .03 \\
0.18 \pm .02\end{array}$ & $0.22 \pm .02$ & & \\
\hline $\begin{array}{r}\text { July } 18 \\
1978 \\
1979\end{array}$ & $\begin{array}{l}0.17 \pm .01 \\
0.10 \pm .01\end{array}$ & $\begin{array}{l}0.22 \pm .02 \\
0.17 \pm .02\end{array}$ & $\begin{array}{l}0.17 \pm .02 \\
0.23 \pm .18\end{array}$ & & \\
\hline $\begin{array}{c}\text { August } 1 \\
1978 \\
1979\end{array}$ & $\begin{array}{l}0.15 \pm .01 \\
0.08 \pm .01\end{array}$ & $\begin{array}{l}0.18 \pm .02 \\
0.16 \pm .01\end{array}$ & $\begin{array}{l}0.16 \pm .02 \\
0.20 \pm .06\end{array}$ & $0.17 \pm .02$ & \\
\hline $\begin{array}{c}\text { Septemb } \\
1978 \\
1979\end{array}$ & $\begin{array}{l}0.17 \pm .02 \\
0.08 \pm .01\end{array}$ & $\begin{array}{l}0.16 \pm .02 \\
0.15 \pm .02\end{array}$ & $\begin{array}{l}0.19 \pm .01 \\
0.18 \pm .02\end{array}$ & $0.20 \pm .03$ & \\
\hline $\begin{array}{c}\text { October } \\
1978 \\
1979\end{array}$ & $0.08 \pm .02$ & $0.14 \pm .03$ & $\begin{array}{l}0.26 \pm .05 \\
0.18 \pm .02\end{array}$ & $0.19 \pm .05$ & $0.21 \pm .02$ \\
\hline $\begin{array}{c}\text { October } \\
1978 \\
1979\end{array}$ & $0.09 \pm .02$ & & $0.14 \pm .02$ & $0.22 \pm .07$ & $0.26 \pm .06$ \\
\hline
\end{tabular}




\section{Discussion}

Two years of clipping at boot, emergence, flowering, and seed formation produced significant reductions in ADF, plus increases in $\mathrm{CP}, \mathrm{Ca}$, and $\mathrm{P}$ on 26 October compared to nondefoliated plants. Other than for $\mathrm{Ca}$, these altered herbage constituents represent improved forage quality available for fall grazing. These results support the theoretical potential to improve fall forage quality with cattle grazing during spring, which delays plant phenology of subsequent regrowth. This regrowth provides higher forage quality not only because of younger photosynthetic tissue, but also because of increased availability due to the removal of old growth (Laycock and Price 1970). Moreoever, the magnitude of improved forage quality is sufficient to warrant considerable interest for grazing management planning.

The National Research Council (1976) recommended minimum levels of $5.9 \% \mathrm{CP}$, plus $0.18 \%$ for $\mathrm{P}$ and $\mathrm{Ca}$ for mature pregnant cows during fall and winter. Nelson and Leege (1982) utilizing data from Thorne et al. (1976) reported a similar requirement of $5.5 \%$ CP for winter maintenance of pregnant elk body weights under feedlot conditions. Presumably elk also share requirements for $\mathrm{Ca}$ and $P$ similar to cattle. Tables 6 and 8 indicate that $C P$ and $P$ of unclipped foliage on 26 October 1979 equalled or fell below these winter requirements respectively, while CP of plants clipped at emergence, flowering and seed formation all exceeded maintenance levels. Foliar $\mathrm{P}$ of plants clipped at flowering and seed formation similarly exceeded maintenance values for cattle and elk on 26 October 1979 (Table 8).

Based upon these experimental plots, fall forage quality improves with successively later clipping treatments, particularly at emergence, flowering, and seed formation. Regrowth of foliage in plants clipped at boot tended to be lower in nutritive quality than with later clipping treatments, even when compared at equivalent phenological stages. These results suggest that plants defoliated during the boot stage of development still retained a sufficient portion of the growing season to respond similarly to undefoliated plants.

Such generalizations, however, should be extrapolated to other sites with caution. Specifically, the extent of regrowth depends upon availability of soil moisture, with response becoming less pronounced as soil moisture declines. For example, Hedrick et al. (1969) found that vegetative regrowth following grazing by yearling heifers occurred only in 2 of 5 years under the prevailing weather patterns of fall-winter-spring precipitation in eastern Oregon. Similarly, the duration of any improved forage quality may not persist into a second growing season. Uresk and Cline (1976) found no differences in nutritive content of bluebunch wheatgrass at seed development 1 year subsequent to 2 years of moderate spring grazing by cattle.

Morcover, intensity and timing of grazing relative to elevation of apical meristems also influences subsequent forage quality. Hyder and Sneva (1963) reported that complete removal of crested wheatgrass (Agropyron desertorum) (Fisch.) Schult.) growing points produced a higher quality forage with increased proportions of vegetative tillers relative to reproductive culms. Bedell (1973) also illustrated that clipping of crested wheatgrass in June removed a substantial proportion of apical meristems, such that summer and fall regrowth contained nearly $50 \%$ more protein than plants clipped in May, where regrowth herbage consisted of both vegetative tillers and reproductive culms.

Anderson and Scherzinger (1975) postulated that spring grazing can produce increased nutrients per unit area for fall utilization relative to ungrazed forage. Demarchi (1973) provided inferential data to support this hypothesis, as moderately grazed bluebunch wheatgrass sites, dominated by needleandthread (Stipa comata Trin. \& Rupr.), yielded greater crude protein per unit area than did lightly grazed bluebunch wheatgrass sites in excellent range condition. Alternatively, Mcllvanie (1942) reported that total nitrogen in bluebunch wheatgrass declined $53 \%$ when clipped biweekly, while Aldous (1930) concluded that observed increases in forage value of frequently clipped prairie grasses may not compensate for decreased yield and density of the vegetative cover. Similarly, Blaisdell et al. (1952) reported that total quantities of crude protein and phosphorus were greater in unclipped bluebunch wheatgrass because herbage yield exceeded that of unclipped plants.

The value of spring grazing to improve quality of fall forage depends upon total available forage as well as grazing ungulate demand for fall forage. Consequently, even if total nutrients per unit area are decreased relative to ungrazed sites, spring grazing may still be beneficial if sufficient forage quantity remains to satisfy fall forage biomass requirements of wintering ungulates. Moreover, because of spring grazing-ind uced phenological delays, forage quality of the remaining available forage will be improved relative to that of ungrazed sites.

Recommendations for specific management plans utilizing spring grazing to improve fall forage quality must assess carefully the physiological impacts of defoliation at alternative phenological stages. Daer and Willard (1981) cautioned that herbage removal of bluebunch wheatgrass late in the growing season may reduce carbohydrate reserves more than defoliation early in the growing season. McLean and Wikeem (1985a) reported that successively later clipping treatments prior to cessation of leaf growth corresponded with increased mortality, reduced leaf length and depressed forage yields. Plant mortality in the current study similarly increased with later defoliation, while the number of flowering stems/plant was severely reduced by clipping at emergence in 2 successive years (Table 9). Consequently, although fall forage qual-

\section{Table 9. Response of bluebunch wheatgrass to two years of defoliation, measured in May, 1980.}

\begin{tabular}{|c|c|c|c|c|c|}
\hline & \multicolumn{5}{|c|}{ Clipping treatment } \\
\hline & Control & Boot & $\begin{array}{l}\text { Emer- } \\
\text { gence }\end{array}$ & $\begin{array}{l}\text { Flower- } \\
\text { ing }\end{array}$ & $\begin{array}{c}\text { Seed } \\
\text { formation }\end{array}$ \\
\hline $\begin{array}{l}\text { Basal Area } \\
\qquad(\mathrm{cm})\end{array}$ & $101 a^{1}$ & $103 a$ & $109 a$ & $109 a$ & $103 a$ \\
\hline $\begin{array}{l}\text { Stem Weight } \\
\text { (g/plant })\end{array}$ & $584 a$ & $649 a$ & $739 a$ & $656 a$ & $772 a$ \\
\hline $\begin{array}{l}\text { Flowering Stems } \\
\text { (\#/plant) }\end{array}$ & $89 a$ & $10 \mathrm{~b}$ & $4 b$ & $23 \mathrm{c}$ & $14 \mathrm{c}$ \\
\hline $\begin{array}{l}\text { Flowers } \\
\text { (\#/basal area) }\end{array}$ & $1.02 \mathrm{a}$ & $0.12 b$ & $0.10 \mathrm{~b}$ & $0.19 b$ & $0.13 b$ \\
\hline $\begin{array}{c}\text { Mortality (\%) } \\
(\# / \mathbf{n})\end{array}$ & $\begin{array}{l}13 \\
(6 / 45)\end{array}$ & $\begin{array}{c}20 \\
(6 / 30)\end{array}$ & $\begin{array}{c}52 \\
(13 / 25)\end{array}$ & $\begin{array}{c}60 \\
(13 / 20)\end{array}$ & $\begin{array}{c}60 \\
(9 / 15)\end{array}$ \\
\hline
\end{tabular}

${ }^{1}$ Means within rows followed by different letters are significantly different at $p<0.05$.

ity is enhanced most by grazing at successively later phenological stages, such improvements occur at the expense of plant health and reproductive potential. Optimum management recommendations must therefore consider all costs and benefits associated with specific grazing management alternatives.

Such benefits must necessarily include increased utilization of the improved forage during fall and winter, with subsequent gains in animal production. Willms et al. (1979) reported increased deer utilization in spring of pastures grazed heavily by cattle during the previous fall. Alternatively, Skovlin et al. (1983) reported no increased utilization by elk during winter of forage theoretically improved by spring cattle grazing. Indeed, elk use actually declined $28 \%$ following cattle grazing in 1 of 3 years of study. Although the recommendation of Anderson and Scherzinger (1975) was considered plausible by Skovlin et al. (1983), they recommended that light cattle use in spring could be maintained primarily when forage supplies exceeded the winter needs of elk. Smith et al. (1979) identified an additional ecological complexity when they reported that although mule deer diets on winter range grazed the previous fall by domestic sheep contained higher proportions of preferred forage species, the nutritive quality of this forage remained unaffected by sheep grazing activities. Such inconsistencies in research 
results clearly indicate the need for site-specific management recommendations.

The current study verifies the hypothesis of Anderson and Scherzinger (1975) that spring defoliation can increase fall forage quality of bluebunch wheatgrass. These nutritional benefits, however, are best enhanced by defoliation during stages when the plant is most susceptiblc to grazing damage. For example, Mueggler (1975) observed that bluebunch wheatgrass required more than 5 years to recover in vigor from a single defoliation of $50 \%$ just prior to flower stalk formation. Consequently, Anderson and Scherzinger's (1975) recommendation must be implemented judiciously, and is best applied within a rotation that permits rest from grazing every second or third year (McLean and Wikeem 1985b).

\section{Literature Cited}

Aldous, A.E. 1930. Effect of different clipping treatments on the yield and vigor of prairie grass vegetation. Ecology 11:752-759.

Anderson, E. William, and Richard J. Scherzinger. 1975. Improving quality of winter forage for elk by cattle grazing. J. Range Manage. 28:120-125.

Association of Official Analytical Chemists. 1975. Methods of analysis (Wiliam Horwitz, ed.).

Bedell, T.E. 1973. Clipping effects on growth form, yield and nutritive quality of crested wheatgrass in eastern Wyoming. Wyo. Agr. Exp. Sta. Res. J. 76.

Blaisdell, James P., and Joseph F. Pechanec. 1949. Effects of herbage removal at various dates on vigor of bluebunch wheatgrass and arrowleaf balsamroot. Ecology 30:298-305.

Blaisdell, James P., A.C. Wiese, and C.W. Hodgson. 1952. Variations in chemical composition of bluebunch wheatgrass, arrowleaf balsamroot, and associated range plants. J. Range Manage. 5:346-353.

Canada Soil Survey Committee. 1978. The Canadian system of soil classification. Res. Branch, Can. Dep. Agr., Ottawa, Ontario.

Daer, Tom, and E. Earl Willard. 1981. Total nonstructural carbohydrate trends in bluebunch wheatgrass related to growth and phenology. J. Range Manage. 34:377-379.

Demarchi, Dennis A. 1973. Relationship of range quality to range condition in the Chilcotin region, British Columbia. J. Range Manage. 26:345-348.

Hanson, W.R., and L.A. Stoddart. 1940. Effects of grazing upon bunch wheatgrass. J. Amer. Soc. Agron. 32:278-289.

Harry, Kenneth F., and J.B. Wright. 1967. The climate of Vancouver. Can. Dep. Transport. Meteor. Branch Cir. 2985.

Heady, Harold F. 1950. Studies on bluebunch wheatgrass in Montana and height-weight relationships of certain range grasses. Ecol. Monogr. 20:55-81.

Hedrick, D.W., W.M. Moser, A.L. Steninger, and R.A. Long. 1969. Animal performance on crested wheatgrass pastures during May and June, Fort Rock. Oregon. J. Range Manage. 22:277-280.

Hormay, August L. 1970. Principles of rest-rotation grazing and multipleuse land management. USDA Forest Serv. Training Text 4 (2200).

Hyder, D.N. 1971. Morphogenesis and management of perennial grasses in the United States. In: Plant morphogenesis as the basis for scientific management of range resources. Proc. U.S./Aust. Rangel. Panel. USDA Misc. Pub. 1271.
Hyder, D.N., and Forrest A. Sneva. 1963. Morphological and physiological factors affecting the grazing management of crested wheatgrass. Crop. Sci. 3:267-271.

Jensen, Charles H., Arthur D. Smith, and George W. Scotter. 1972. Guidelines for grazing sheep on rangelands used by big game in winter. $J$. Range Manage. 25:346-352.

Laycock, William A., and Donald A. Price. 1970. Factors influencing forage quality. In: Range and wildlife habitat evaluation: A research symposium. USDA Misc. Pub. 114.

Malechek, John C., Kurt J. Kotter, and Charles H. Jensen. 1978. Nutrition and production of domestic sheep managed as manipulators of big game habitat. J. Range Manage. 31:92-96.

Mellvanie, Samuel K. 1942. Carbohydrate and nitrogen trends in bluebunch wheatgrass, Agropyron spicatum, with special reference to grazing influences. Plant Physio. 17:550-557.

McLean, Alastair, and Sandra Wikeem. 1985a. Influence of season and intensity of defoliation on bluebunch wheatgrass survival and vigor in southern British Columbia. J. Range Manage. 38:21-26.

McLean, Alastair, and Sandra Wikeem. 1985b. Defoliation effects on three range grasses. Rangelands 7:61-63.

Metcalfe, Darrel S. 1973. The botany of grasses and legumes. p. 80-97. In: Heath, Maurice E., Darrel S. Metcalfe, and Robert E. Barnes (eds.). Forages, the science of grassland agriculture, 3rd ed. Iowa State Univ. Press.

Mueggler, W.F. 1975. Rate and pattern of vigor recovery in Idaho fescue and bluebunch wheatgrass. J. Range Manage. 28:198-204.

National Research Council. 1976. Nutrient requirements of domestic animals; No. 4, Beef Cattle, 5th ed., National Academy of Sciences, Washington, D.C.

Nelson, Jack R., and Thomas A. Leege. 1982. Nutritional requirements and food habits. p. 323-362. In: Thomas, Jack Ward and Toweill, Dale F. (eds.). Elk of North America; Ecology and management.

Rickard, W.H., D.W. Uresk, and J.F. Cline. 1975. Impact of cattle grazing on three perennial grasses in south-central Washington J. Range Manage. 28:108-112.

Skovln, Jon M., Paul J. Edgerton, and Burt R. McConnell. 1983. Elk use of winter range as affected by cattle grazing, fertilizing, and burning in southeastern Washington. J. Range Manage. 36:184-189.

Smith, Michael A., John C. Malachek, and Kenneth O. Fulgham. 1979. Forage selection by mule decr on winter range grazed by sheep in spring. J. Range Manage. 32:40-45.

Steele, Robert G.D., and James H. Torrie. 1980. Principles and procedures of statistics. McGraw-Hill Inc.

Stoddart, L.A. 1946. Some physical and chemical responses of Agropyron spicatum to herbage removal at various seasons. Utah Agr. Exp. Sta. Bull. 324.

Thorne, E. Tom, Ron E. Dean, and William G. Hepworth. 1976. Nutrition during gestation in relation to successful reproduction in elk. J. Wildl. Manage. 40:330-335.

Uresk, Daniel W., and John F. Cline. 1976. Mineral composition of three perennial grasses in a shrub-steppe community in south-central Washington. J. Range Manage. 19:255-256.

Willms, W., A. McLean, R. Tucker, and R. Ritcey. 1979. Interactions between mule deer and cattle on big sagebrush range in British Columbia. J. Range Manage. 32:299-304. 